\title{
Linear-Quadratic Stochastic Pursuit-Evasion Games
}

\author{
Arunabha Bagchi and Geert Jan Olsder \\ Department of Applied Mathematics, Twente University of Technology, P.O. Box 217, 7500 AE \\ Enschede, The Netherlands \\ Communicated by A. Bensoussan
}

\begin{abstract}
A linear-quadratic differential game in which the system state is affected by disturbance and both players have access to different measurements is solved. The problem is first converted to an optimization problem in infinite-dimensional state space and then solved using standard techniques. For convenience, " $L_{2}$-white noise" instead of "Wiener process" setup is used.
\end{abstract}

\section{Introduction}

A two-person pursuit-evasion differential game is considered in which the system state is affected by noise. Both the pursuer, $P_{p}$, and the evader, $P_{e}$, know the system characteristics and have access to noisy measurements which are not necessarily the same. In traditional control and differential game problems, the assumption is always made that information is centralized; that is, the player(s) have access to complete information. This no longer holds in the present context. Player $P_{p}$ does not know the measurements of $P_{e}$, and there is no reason for $P_{e}$ to make that information available to $P_{p}$, and vice versa.

We shall consider linear dynamics and quadratic payoff, because they are analytically tractable and also because they arise in the perturbation analysis of nonlinear pursuit-evasion game problems. There are essentially three possibilities as far as measurements are concerned; namely, no measurement, exact measurements or noisy measurements. This leads to nine different cases for two-player games, of which six are essentially different, by symmetry. Except for the case in which both players have noisy measurements, all other cases have been solved; see for instance [3], [4], [5], [9].

For the case of noisy measurements for both players, a purely formal solution has been given by Willman [10], which motivated our present study. Incidentally, the solution to the particular case in which the same observation is available to both the players is well known [1]. The method employed there cannot be 
extended to the case in which the players have different noisy measurements because each player has only imperfect knowledge of the information available to his opponent. Here it is assumed that each player has perfect recall with respect to his own information but does not have access to the information of the other player. In terms of information structures [7], the information structure is not nested.

We solve the linear-quadratic pursuit-evasion game with possibly different noisy measurements for the players under the assumption that the decisions will be linear functionals of the available measurements. We give sufficient conditions that ensure solution of the problem in this class of linear strategies. This, in turn, is shown to be the unique saddle point in a much broader class of control strategies. To solve the problem in the linear class mentioned above, we introduce new state variables with values in a Hilbert space and convert the original problem to an optimization problem with an infinite-dimensional state space. To facilitate mathematical computations, we work exclusively in the Hilbert space setting, and therefore, we use the "white noise" setup of Balakrishnan [2], as opposed to the conventional Wiener process approach. For linear problems, these two approaches are identical. We solve the problem by first fixing the strategy of the evader, solving the control problem involving the strategy of the pursuer, and then repeating this procedure with the roles of the players interchanged.

In section 2 we formulate the problem, give sufficient conditions ensuring the existence of saddle points in the class of linear strategies, and point out that this is also the unique saddle point in a broader class of strategies. In section 3 we introduce new state variables in infinite dimension and convert the original problem to optimization problems with infinite-dimensional state spaces. In section 4 we solve the original problem, and in section 5 we discuss special cases in which solutions are known already, including a comparison with the formal results obtained in [10]. Finally, in section 6 we study the question of solving the set of coupled partial integro-differential equations that characterize our solution.

\section{Problem Formulation}

We use the $L_{2}$-theory of white noise for modeling our noise process. The details can be found in [2]. The differential equation describing the evolution of the system in state space is

$$
\begin{aligned}
\dot{x}(t ; \omega)= & A(t) x(t ; \omega)+B_{p}(t) u_{p}(t ; \omega)+B_{e}(t) u_{e}(t ; \omega) \\
& +F(t) n(t ; \omega) \quad 0 \leqslant t \leqslant T \\
x(0 ; \omega)= & x_{0}
\end{aligned}
$$

where

$n(. ; \omega) ; \omega \in L_{2}^{q}[0, T]$ is a white noise in $L_{2}^{q}[0, T]$;

$x(t ; \omega)$ is an $n$-vector denoting the state;

$u_{p}(t ; \omega)$ is an $r_{p}$-vector denoting the strategy of the pursuer;

$u_{e}(t ; \omega)$ is an $r_{e}$-vector denoting the strategy of the evader. 
The matrices $A(t), B_{p}(t), B_{e}(t)$, and $F(t)$ have appropriate dimensions. The initial state $x_{0}$ is assumed to be normal, which, without loss of generality, we take to be of zero mean:

$$
x_{0} \sim N\left(\bar{x}_{0}, P_{0}\right), \quad \bar{x}_{0}=0
$$

The observations available to the pursuer and evader are, respectively:

$$
\begin{aligned}
& y_{p}(t ; \omega)=C_{p}(t) x(t ; \omega)+G_{p}(t) n(t ; \omega), \\
& y_{e}(t ; \omega)=C_{e}(t) x(t ; \omega)+G_{e}(t) n(t ; \omega)
\end{aligned}
$$

where $y_{p}$ and $y_{e}$ are $m_{p}-$ and $m_{e}$-vectors, respectively.

We assume the strategies $u_{p}(t ; \omega)$ and $u_{e}(t ; \omega)$ at time $t$ to be linear functionals of the observations $y_{p}(s ; \omega), 0 \leqslant s \leqslant t$ and $y_{e}(s ; \omega), 0 \leqslant s \leqslant t$, respectively:

$$
\begin{aligned}
& u_{p}(t)=\int_{0}^{t} N_{p}(t, \tau) y_{p}(\tau) d \tau, \\
& u_{e}(t)=\int_{0}^{t} N_{e}(t, \tau) y_{e}(\tau) d \tau,
\end{aligned}
$$

where the kernels $N_{p, e}(t, \tau)$ are partially differentiable w.r.t. $\tau$ and $(\partial / \partial \tau) N_{p, e}(t, \tau)$ is square integrable on the triangle $0 \leqslant \tau \leqslant t \leqslant T$. Within this class of control strategies the problem is to find

$$
\sup _{u_{e}} \inf E\{J\}
$$

where

$$
\begin{aligned}
J= & \frac{1}{2}\left\{\left\langle x(T ; \omega), Q_{f} x(T ; \omega)\right\rangle+\int_{0}^{T}[\langle x(t ; \omega), Q(t) x(t ; \omega)\rangle\right. \\
& \left.\left.+\left\langle u_{p}(t ; \omega), R_{p}(t) u_{p}(t ; \omega)\right\rangle-\left\langle u_{e}(t ; \omega), R_{e}(t) u_{e}(t ; \omega)\right\rangle\right] d t\right\}
\end{aligned}
$$

where $R_{p}(t), R_{e}(t)$ are positive definite for a.e. $t$ on $[0, T]$. At this moment it is not known whether optimal strategies exist in the admissible class as given in (2.5).

It is assumed that both players know and have perfect recall as to the system characteristics $\Sigma$ :

$$
\Sigma \triangleq\left\{A, B_{p}, B_{e}, C_{p}, C_{e}, F, G_{p}, G_{e}, P_{0}, \bar{x}_{0}, Q_{f}, Q, R_{p}, R_{e}\right\}
$$

and also as to their own past measurements and controls. The distribution of $x_{0}$ is independent of process and measurement noise. Process and measurement noise are not necessarily independent, contrary to the assumption made in [10]. 
We use a somewhat unconventional model for the noise process. This is done for convenience. In solving the problem stated in this section, we have to extend the state space to infinite dimension, as explained in the next section. In our present formulation, this extended state is an element of a Hilbert space. The criterion $J$, when rewritten in the new state space, has the form of a quadratic functional in a Hilbert space and $L Q G$ theory in Hilbert space can be routinely applied. On the other hand, if we would use the standard Wiener process formulation, the extended state would be an element of the space of continuous functions on $[0, T]$, which is a Banach space. The criterion $J$, when rewritten in the new state space, would be a functional on a Banach space. This difficulty may not be insurmountable, but it is clearly unnecessary. We do not claim that our approach is the only possible one for solving the present problem.

\section{Reformulation in Infinite-Dimensional State Space}

Superficially, one might be tempted to try to solve this problem by the standard techniques used in stochastic regulator problems with imperfect observations. Suppose that $u_{p}(t)$ is a (linear) function of $\hat{x}(t)$, where $\hat{x}(t)=E\left\{x(t) \mid Y_{p}(t)\right\}$, where $Y_{p}(t)$ is the weak random variable (see [2]) $\left\{y_{p}(s), 0 \leqslant s \leqslant t\right\}$. In order to calculate $\hat{x}(t), P_{p}$ needs the knowledge of $u_{e}(s), 0 \leqslant s \leqslant t$ and hence of $y_{e}(s)$, $0 \leqslant s \leqslant t$, which he does not have. Therefore, $P_{p}$ has to estimate $y_{e}(s), 0 \leqslant s \leqslant t$. This estimate, however, will depend on the estimate $P_{e}$ made of $y_{p}(s), 0 \leqslant s \leqslant t$, and so on. We therefore abandon this approach.

Let $\mu$ be the Lebesgue-Stieltjes measure on $[0, T]$ which is the Lebesgue measure there together with unit masses concentrated at $t=0$ and $t=T$. Let $L_{2}(\mu)$ denote the space of Lebesgue measurable functions $f$ mapping $[0, T]$ into $\mathbb{R}^{p}$ such that $\int_{0}^{T}\|f\|^{2} d \mu<\infty,\|\cdot\|$ denoting norm in $\mathbb{R}^{p}$. This is a Hilbert space under the inner product

$$
\langle f, g\rangle=\int_{0}^{T} f^{*}(s) g(s) d s+f^{*}(0) g(0)+f^{*}(T) g(T) .
$$

Introduce new variables $\pi(t), \eta_{p}(t), \eta_{e}(t)$ with values in $L_{2}^{n}(\mu), L_{2}^{m_{p}}(\mu), L_{2}^{m_{e}}(\mu)$, respectively:

$$
\begin{aligned}
{[\pi(t ; \omega)](s) } & =x(s \Lambda t ; \omega) \\
{\left[\eta_{p}(t ; \omega)\right](s) } & =\int_{0}^{s \Lambda t} G_{p}(\tau) n(\tau ; \omega) d \tau \\
{\left[\eta_{e}(t ; \omega)\right](s) } & =\int_{0}^{s \Lambda t} G_{e}(\tau) n(\tau ; \omega) d \tau
\end{aligned}
$$

where $s \Lambda t=\min \{t, s\}$. We convert our original problem to solving two optimal control problems. First, assume that $N_{e}(t, \tau)$ is fixed and then the optimal $u_{p}(t ; \omega)$ is expressed in terms of $N_{e}(t, \tau)$. Fortunately, this optimal $u_{p}$ turns out to be of the form as given in (2.5) and hence $N_{p}$ can be expressed as a function of $N_{e}$. Subsequently, the roles of $u_{p}$ and $u_{e}$ are interchanged. Thus we have obtained two 
implicit equations for the two unknown functions $N_{p}$ and $N_{e}$. The assumption about the class of admissible strategies being linear in the observations $((2.5))$ was simply a bet, which turns out to be a winning one, provided that the two implicit equations have a solution. Suppose now that $N_{e}(t, \tau)$ is given. Then $u_{e}(t, \omega)$ can be written as

$$
\begin{aligned}
u_{e}(t ; \omega)= & \int_{0}^{t} N_{e}(t, \tau)\left[C_{e}(\tau) x(\tau ; \omega)+G_{e}(\tau) n(\tau ; \omega)\right] d \tau \\
= & \int_{0}^{t} N_{e}(t, \tau) C_{e}(\tau)[\pi(t ; \omega)](\tau) d \tau+N_{e}(t, t)\left[\eta_{e}(t ; \omega)\right](T) \\
& -\int_{0}^{t}\left(\frac{d}{d \tau} N_{e}(t, \tau)\right)\left[\eta_{e}(t ; \omega)\right](\tau) d \tau
\end{aligned}
$$

In the new state space $L_{2}^{n}(\mu) \times L_{2}^{m_{e}}(\mu)$, the model can be expressed as

$$
\begin{aligned}
\frac{d}{d t}\left[\begin{array}{c}
\pi(t) \\
\eta_{e}(t)
\end{array}\right]= & {\left[\begin{array}{c|c}
\mathscr{Q}(t) & \mathscr{B}(t) \\
\hdashline 0 & 0
\end{array}\right]\left[\begin{array}{c}
\pi(t) \\
\eta_{e}(t)
\end{array}\right] } \\
& +\left[\begin{array}{c}
\mathscr{B}_{p}(t) \\
-0
\end{array}\right] u_{p}(t)+\left[\begin{array}{c}
\mathscr{G}(t) \\
\overline{\mathcal{G}_{e}}(t)
\end{array}\right] n(t)
\end{aligned}
$$

and the initial condition becomes

$$
\left[\begin{array}{c}
\pi(0) \\
\eta_{e}(0)
\end{array}\right] \sim N\left(0,\left[\begin{array}{c|c}
\mathscr{P}_{0} & 0 \\
\hdashline 0 & 0 \\
0 & 0
\end{array}\right]\right)
$$

The measurement equation can be written as

$$
y_{p}(t)=\left[\begin{array}{l:l}
e_{p}(t) & 0
\end{array}\right]\left[\begin{array}{c}
\pi(t) \\
\eta_{e}(t)
\end{array}\right]+G_{p}(t) n(t) .
$$

The operators $\mathscr{Q}(t), \mathscr{B}(t), \mathscr{B}_{p}(t), \mathcal{G}_{e}(t), \mathscr{F}(t), \mathcal{C}_{p}(t), \mathscr{P}_{0}$ are given in Appendix A. Substitution of $u_{e}(t)$ from (3.4) enables us to express $J$ in terms of the new variables as

$$
\begin{aligned}
& J=\frac{1}{2}\left\{\left\langle\left[\begin{array}{c}
\pi(T) \\
\eta_{e}(T)
\end{array}\right],\left[\begin{array}{c|c}
\chi_{f}^{*} \chi_{f} & 0 \\
\hdashline 0 & - \\
\hdashline
\end{array}\right]\left[\begin{array}{c}
\pi(T) \\
\eta_{e}(T)
\end{array}\right]\right\rangle\right.
\end{aligned}
$$

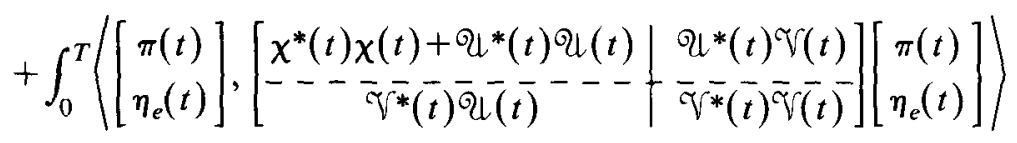

$$
\begin{aligned}
& \left.+\int_{0}^{T}\left\langle u_{p}(t), R_{p}(t) u_{p}(t)\right\rangle d t\right\} \text {. }
\end{aligned}
$$


The operators $\chi, \chi_{f}, \mathcal{Q}, \widetilde{V}$ are also given in Appendix A. Player $P_{p}$ wants to choose $u_{p}(t)$ as a function of the measurement (3.7) so as to minimize (3.8) subject to (3.5) and (3.6). This is the stochastic regulator problem in the Hilbert space setting, which has been solved in [2] with $\mathscr{Q}(t), \mathscr{B}(t)$ being unbounded operators. Our operators, on the contrary, are bounded. This, however, only makes our problem easier to handle mathematically and the theory developed in [2] can be used without alteration.

\section{Solution}

We apply the theory of stochastic regulator problem in Hilbert space to the problem described in section 3, which minimizes (3.8) - subject to (3.5) and (3.6) in the class of strategies $u_{p}(t)$ which are linear functions of $y_{p}(s), 0 \leqslant s \leqslant t$, as given in (3.7). The optimal $u_{p}(t)$ is

$$
u_{p}^{0}(t)=-R_{p}^{-1}(t)\left[\mathscr{B}_{p}^{*}: 0\right] \mathscr{K}(t)\left[\begin{array}{c}
\hat{\pi}(t) \\
\hdashline \hat{\eta}_{e}(t)
\end{array}\right],
$$

where the operator $\mathscr{K}(t)$, the control gain, satisfies

$$
\begin{aligned}
& \dot{K}(t)=-\left[\begin{array}{c:c}
\mathbb{Q}^{*}(t) & 0 \\
\hdashline \mathscr{G}^{*}(t) & 0
\end{array}\right] \mathscr{K}(t)-\mathscr{K}(t)\left[\begin{array}{c:c}
\mathscr{Q}(t) & \mathscr{B}(t) \\
\hdashline 0 & 0
\end{array}\right]
\end{aligned}
$$

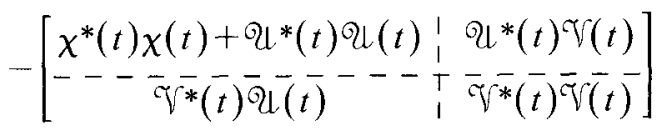

$$
\begin{aligned}
& +\mathscr{K}(t)\left[\begin{array}{c}
\mathscr{B}_{p}(t) \\
\hdashline 0
\end{array}\right] R_{p}^{-1}(t)\left[\mathscr{B}_{p}^{*}: 0\right] \mathscr{K}(t) ; \mathscr{K}(T)=\left[\begin{array}{c:c}
\chi_{f}^{*} \chi_{f} & 0 \\
\hdashline 0 & 0
\end{array}\right] \text {. }
\end{aligned}
$$

Define

$$
\Gamma(t) \triangleq\left(\mathscr{P}(t)\left[\begin{array}{c}
\mathcal{C}_{p}^{*}(t) \\
-0-
\end{array}\right]+\left[\begin{array}{c}
\mathscr{F}(t) \\
\mathcal{G}_{e}(t)
\end{array}\right] G_{p}^{*}(t)\right)\left(G_{p}(t) G_{p}^{*}(t)\right)^{-1},
$$

where $\mathscr{P}(t)$ satisfies

$$
\begin{aligned}
& \dot{\mathscr{P}}(t)=\left[\begin{array}{c:c}
\mathscr{Q}(t) & \mathscr{P}(t) \\
\hdashline 0 & 0
\end{array}\right] \mathscr{P}(t)+\mathscr{P}(t)\left[\begin{array}{c:c}
\mathbb{Q} *(t) & 0 \\
\hdashline \mathscr{G} *(t) & 0
\end{array}\right]
\end{aligned}
$$

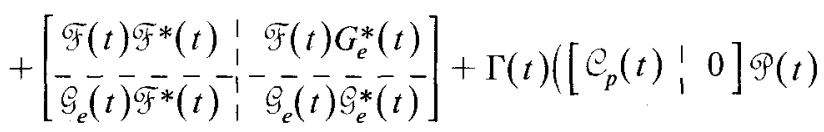

$$
\begin{aligned}
& \left.+G_{p}(t)\left[\mathscr{F}^{*}(t): \mathcal{G}_{e}^{*}(t)\right]\right), \quad \mathscr{P}(0)=\left(\begin{array}{c:c:c}
\mathscr{P}_{0} & 0 \\
\hdashline 0 & 0
\end{array}\right) .
\end{aligned}
$$




\section{Defining}

$$
\begin{aligned}
& \Omega(t)=\left[\begin{array}{c:c}
\mathscr{Q}(t) & \mathscr{B}(t) \\
\hdashline 0 & 0
\end{array}\right]-\Gamma(t)\left[\mathcal{C}_{p}(t): 0\right] \\
& -\left[\begin{array}{c}
\mathscr{B}_{p}(t) \\
\hdashline 0
\end{array}\right] R_{p}^{-1}(t)\left[\mathscr{B}_{p}^{*}(t): 0\right] \mathscr{K}(t),
\end{aligned}
$$

then the estimate of the state can be written as

$$
\frac{d}{d t}\left[\begin{array}{c}
\hat{\pi}(t) \\
-\hat{\eta}_{e}(t)
\end{array}\right]=\Omega(t)\left[\begin{array}{c}
\hat{\pi}(t) \\
-\hat{\eta}_{e}(t)
\end{array}\right]+\Gamma(t) y_{p}(t) .
$$

The fundamental operator of $\Omega(t)$ defined in [11], is denoted by $\Psi$

$$
\frac{d}{d t} \Psi(t, \sigma)=\Omega(t) \Psi(t, \sigma) ; \Phi(\sigma, \sigma)=I .
$$

\section{Defining}

$$
\varsigma(t, \tau)=\Psi(t, \tau) \Gamma(\tau),
$$

then $\mathcal{\delta}$ satisfies

$$
\frac{d}{d t} \mathfrak{S}(t, \sigma)=\Omega(t) \mathcal{S}(t, \sigma) ; \quad \delta(\sigma, \sigma)=\Gamma(\sigma),
$$

and we have

$$
\left[\begin{array}{l}
\hat{\pi}(t) \\
\hat{\eta}_{e}(t)
\end{array}\right]=\int_{0}^{t} \delta(t, \sigma) y_{p}(\sigma) d \sigma .
$$

Using (2.6), (4.1), and (4.6), we get the following solution for $u_{p}(t)$, and hence for $N_{p}(t, \tau)$

$$
\begin{aligned}
& u_{p}(t)=\int_{0}^{t} N_{p}(t, \tau) y_{p}(\tau) d \tau \\
& =-\int_{0}^{t} R_{p}^{-1}(t)\left[\mathscr{B}_{p}^{*}(t), 0\right] \mathcal{K}(t) \delta(t, \tau) y_{p}(\tau) d \tau .
\end{aligned}
$$

The right-hand side of (4.7) obviously depends on $N_{e}$, and thus (4.7) gives the first relation connecting $N_{P}$ and $N_{e}$. Here we notice that the solution obtained is indeed of the form (2.5), as indicated already in the previous section. Interchanging the roles of the players, another relation between $N_{p}$ and $N_{e}$ is obtained. These two relations in principle determine the functions $N_{p}$ and $N_{e}$.

Remark. In section 6 it will be proved that provided certain conditions on $\Sigma$ are satisfied, the optimal $N_{p, e}(t, \tau)$ exist. Let us assume this to be true for a moment. 
Let the corresponding optimal control functions be denoted by $u_{p}^{*}, u_{e}^{*}$ and the criterion $E\{J\}$ by $V\left(u_{p}, u_{e}\right)$. Then

$$
V\left(u_{p}^{*}, u_{e}\right) \leqslant V\left(u_{p}^{*}, u_{e}^{*}\right) \leqslant V\left(u_{p}, u_{e}^{*}\right) .
$$

Obviously inequalities (4.7a) hold for $u_{p, e}$ linear in the past observations. This class of strategies can be broadened, however. The first inequality of (4.7a) holds for all $u_{e}$ for which the system of equations

$$
\begin{aligned}
\dot{x} & =A x+B_{p} u_{p}^{*}+B_{e} u_{e}+F n, \\
u_{p}^{*} & =\int_{0}^{t} N_{p}(t, \tau) y_{p}(\tau) d \tau, \\
y_{p} & =C_{p} x+G_{p} n, \\
u_{e} & =\text { nonanticipative functional of } y_{e}(s), \quad 0 \leqslant s<t, \\
y_{e} & =C_{e} x+G_{e} n
\end{aligned}
$$

has a unique solution on $[0, T]$. Something similar holds w.r.t the second inequality in (4.7a).

Equation (4.7) essentially solves the problem. The ultimate goal, however, is to find the solution in terms of the system characteristics $\Sigma$, see (2.8). In Appendix $\mathrm{B}$ the representation for $\mathcal{S}, \mathscr{P}$, and $\mathcal{K}$ has been derived. It is shown that for $x \in \mathbb{R}^{m_{p}}, f, g, \in L_{2}^{n}(\mu) \times L_{2}^{m_{e}}(\mu)$,

$$
\begin{aligned}
\delta(t, \tau) x & =g, \text { where } g(s)=S(t, \tau, s) x, \quad 0 \leqslant s \leqslant T ; \\
\mathcal{P}(t) f & =g,
\end{aligned}
$$

where

$$
\begin{aligned}
g(s) & =\int_{0}^{T} P(t, s, \sigma) f(\sigma) d \sigma+p(t, s) f(0)+\bar{p}(t, s) f(T) ; \quad 0 \leqslant s \leqslant T ; \\
\mathscr{K}(t) f & =g,
\end{aligned}
$$

where

$$
g(s)=\int_{0}^{T} K(t, s, \sigma) f(\sigma) d \sigma+\underline{k}(t, s) f(0)+\bar{k}(t, s) f(T), \quad 0 \leqslant s \leqslant T .
$$

Matrix $S$ is partitioned as $\left[\begin{array}{c}S_{1} \\ -S_{2}\end{array}\right]$. The matrices $P, \underline{p}, \bar{p}, K, \underline{k}, \bar{k}$ are partitioned in four blocks. Now it follows from (4.7) that $N_{p}$ can be written as:

$$
N_{p}(t, \tau)=-R_{p}^{-1}(t) B_{p}^{*}(t)\left\{h(T)+\int_{t}^{T} h(s) d s\right\}, \quad 0 \leqslant \tau \leqslant t,
$$


where

$$
\begin{aligned}
h(s)=\sum_{i=1}^{2}[ & \int_{0}^{T} K_{1 i}(t, s, \sigma) S_{i}(t, \tau, \sigma) d \sigma+\underline{k}_{1 i}(t, s) S_{i}(t, \tau, 0) \\
& \left.\quad \bar{k}_{1 i}(t, s) S_{i}(t, \tau, T)\right] .
\end{aligned}
$$

The relationship of $K_{i j}, S_{i}, \underline{k}_{i j}, \bar{k}_{i j}, P_{i j}, \underline{p}_{i j}, \bar{p}_{i j}$ with respect to the matrices of the set $\Sigma$ is given in Appendix $\bar{C}$.

\section{Some Special Cases}

Three special cases will be considered: (1) the standard regulator problem where only one player is present; (2) the measurement and state noises are independent; comparison with Willman's results; and (3) the observations for both players are the same.

\subsection{One-Person Differential Game}

We assume $B_{e}(t) \equiv 0, C_{e}(t) \equiv 0, u_{e}(t) \equiv 0, G_{e}(t) \equiv 0$. First the $K$-functions, as given by differential equations in Appendix $C$, are considered. From the differential equations for $K_{11}$ and $\bar{k}_{11}$ and corresponding final conditions it follows that

$$
K_{11}(t, s, \sigma) \equiv 0, \bar{k}_{11}(t, s) \equiv 0,0 \leqslant t \leqslant T, \quad 0 \leqslant s, \sigma<T \text {. }
$$

For $\bar{k}_{11}(t, T)$ the following differential equation remains:

$$
\begin{aligned}
& \frac{d \bar{k}_{11}(t, T)}{d t}=-A^{*}(t) \bar{k}_{11}(t, T)-\bar{k}_{11}(t, T) A(t)-Q(t) \\
&+\bar{k}_{11}(t, T) B_{p}(t) R_{p}^{-1}(t) B_{p}^{*}(t) \bar{k}_{11}(t, T), \\
& \bar{k}_{11}(T, T)=Q_{f} .
\end{aligned}
$$

The other $K$ - and $k$-functions are all identically zero. Also w.r.t. the $P$ equations we get great simplifications. It is easily seen that

$$
P_{i 2}(t, s, \sigma) \equiv 0, \bar{p}_{i 2}(t, s) \equiv 0, \underline{p}_{i 2}(t, s) \equiv 0,0 \leqslant t, s, \sigma \leqslant T, \quad i=1,2,
$$

and also that

$$
\bar{p}_{11}(t, s) \equiv p_{11}(t, s, T), \underline{p}_{11}(t, s) \equiv P_{11}(t, s, 0) .
$$


We will only be interested in the behavior of $\bar{p}_{11}(t, T)$;

$$
\begin{gathered}
\frac{d \bar{p}_{11}(t, T)}{d t}=A(t) \bar{p}_{11}(t, T)+\bar{p}_{11}(t, T) A^{*}(t)+F(t) F^{*}(t) \\
+\gamma(t)\left(C_{p}(t) \bar{p}_{11}(t, T)+G_{p}(t) F^{*}(t)\right) ; \bar{p}_{11}(0, T)=P_{0},
\end{gathered}
$$

where

$$
\gamma(t)=\left(\bar{p}_{11}(t, T) C_{p}^{*}(t)+F(t) G_{p}^{*}(t)\right)\left(G_{p}(t) G_{p}^{*}(t)\right)^{-1} .
$$

Equation (4.10) is in this case reduced to

$$
h(s)=\bar{k}_{11}(t, s) S_{1}(t, \tau, T)
$$

which leads to

$$
N_{p}(t, \tau)=-R_{p}^{-1}(t) B_{p}^{*}(t) \bar{k}_{11}(t, T) S_{1}(t, \tau, T) .
$$

W.r.t. the $S$-equations the following simplifications are obtained:

$$
\begin{aligned}
& \frac{\partial S_{1}(t, \tau, T)}{\partial t}=A(t) S_{1}(t, \tau, T)+B_{p}(t) N_{p}(t, \tau)-\gamma(t) C_{p}(t) S_{1}(t, \tau, T), \\
& S_{1}(\tau, \tau, T)=\gamma(\tau) .
\end{aligned}
$$

The functions $S_{1}(t, \tau, s)$ for $s<T$ and $S_{2}(t, \tau, s)$ do not appear in the solution, which is given by equations (5.1)-(5.4) and which equals the solution of the standard regulator problem.

\subsection{Comparison with Willman's Solution}

Willman [10] assumed $A(t) \equiv 0, Q(t) \equiv 0, F(t) G_{p}^{*}(t) \equiv 0, F(t) G_{e}^{*}(t) \equiv 0$, and $G_{p}(t) G_{e}^{*}(t) \equiv 0$. In order to be able to compare the results, we make these assumptions also (in this subsection). Defining

$$
\begin{aligned}
& S_{p}(t, \tau) \triangleq S_{1}(t, \tau, T), \\
& L(t, \tau, \sigma) \triangleq C_{e}(\sigma) S_{1}(t, \tau, \sigma)+\frac{\partial S_{2}(t, \tau, \sigma)}{\partial \sigma}, \\
& P(t) \triangleq P_{11}(t, T, T), \\
& M(t, \sigma) \triangleq P_{11}(t, T, \sigma) C_{e}^{*}(\sigma)+\frac{\partial P_{12}(t, T, \sigma)}{\partial \sigma}, \\
& V(t, \rho, \sigma) \triangleq C_{e}(\rho) P_{11}(t, \rho, \sigma) C_{e}^{*}(\sigma)+\frac{\partial P_{21}(t, \rho, \sigma)}{\partial \rho} C_{e}(\rho) \\
& \quad+C_{e}(\rho) \frac{\partial P_{12}(t, \rho, \sigma)}{\partial \sigma}+\frac{\partial P_{22}(t, \rho, \sigma)}{\partial \rho \partial \sigma},
\end{aligned}
$$


then these newly defined functions satisfy (the " $t$ " argument for functions of $t$ only has been suppressed):

$$
\begin{aligned}
\frac{\partial S_{p}(t, \tau)}{\partial t}= & \tilde{B}_{e} \int_{0}^{t} \bar{N}_{e}(t, \sigma) L(t, \tau, \sigma) d \sigma+\tilde{B}_{p} \bar{N}_{p}(t, \tau) \\
& +P C_{p}^{*}\left(G_{p} G_{p}^{*}\right)^{-1} C_{p} S_{p}(t, \tau), \\
S_{p}(t, t)= & P C_{p}^{*}\left(G_{p} G_{p}^{*}\right)^{-1} ; \\
\frac{\partial L(t, \tau, \sigma)}{\partial t}= & -M^{*}(t, \sigma) C_{p}^{*}\left(G_{p} G_{p}^{*}\right)^{-1} C_{p} S_{p}(t, \tau), \quad 0 \leqslant \sigma<t ; \\
L(t, t, \sigma)= & M^{*}(t, \sigma) C_{p}^{*}\left(G_{p} G_{p}^{*}\right)^{-1} ; \\
\frac{d P}{d t}= & F F^{*}+\tilde{B}_{e} \int_{0}^{t} \bar{N}_{e}(t, \rho) M^{*}(t, \rho) d \rho \\
& +\int_{0}^{t} M(t, \rho) \bar{N}_{e}^{*}(t, \rho) d \rho, \tilde{B}_{e}-P C_{p}^{*}\left(G_{p} G_{p}^{*}\right)^{-1} C_{p} P, \\
P(0)= & P_{0} ; \\
\frac{\partial M(t, \sigma)}{\partial t}= & -P C_{p}^{*}\left(G_{p} G_{p}^{*}\right)^{-1} C_{p} M(t, \sigma)+\tilde{B}_{e} \int_{0}^{t} \bar{N}_{e}(t, \rho) V(t, \rho, \sigma) d \rho ; \quad 0 \leqslant \sigma<t ; M(t, t)=P C_{e}^{*} ; \\
\frac{\partial V(t, \rho, \sigma)}{\partial t}= & -M^{*}(t, \rho) C_{p}^{*}\left(G_{p} G_{p}^{*}\right)^{-1} C_{p} M(t, \sigma), 0 \leqslant \rho, \sigma<t, \\
V(t, \rho, t)= & V(t, t, \rho)=M(t, \rho) C_{e}^{*}(\rho),
\end{aligned}
$$

where $\bar{N}_{p}, \bar{N}_{e}$ have been defined as

$$
N_{p}(t, \tau)=R_{p}^{-1}(t) B_{p}^{*}(t) \bar{N}_{p}(t, \tau) ; N_{e}(t, \tau)=R_{e}^{-1}(t) B_{e}^{*}(t) \bar{N}_{e}(t, \tau),
$$

and where $\tilde{B}_{e}$ and $\tilde{B}_{p}$ have been defined in Appendix C. Comparing these differential equations with those of Willman, we see that

$$
\begin{aligned}
& S_{p}(t, \tau) \quad \text { (present paper) }=K_{p}(t, \tau) \quad \text { (Willman); } \\
& L(t, \tau, \sigma)(" \text { " " })=L(t, \tau, \sigma)(" \text { " ); } \\
& P(t) \quad(" \prime \prime)=P(t) \quad(" \prime) \text {; } \\
& V(t, \rho, \sigma)(" \text { " " ) }=N(t, \rho, \sigma)(" \text { "), }
\end{aligned}
$$

where it is assumed that

$$
M(t, \sigma)(\text { present paper })=M(t, \sigma)(\text { Willman }) .
$$

The differential equations for $M(t, \sigma)$, however, do not agree. W.r.t. the nomenclature of the present paper, Willman's differential equation for $M(t, \sigma)$ 
has at the right-hand side an additional term, viz.

$$
\tilde{B}_{e}(t) \bar{N}_{e}(t, \sigma) G_{e}(\sigma) G_{e}^{*}(\sigma)
$$

The reason for this disparity is unclear to the authors. It could be latent in some of the limiting operations in [10]. It is conjectured, however, that it is due to the particular structure of measurements in the discrete time setup in [10]. At stage $j$ the measurements up to and including stage $j$, i.e., $y_{0}, \ldots, y_{j}$ are available for estimates instead of only the measurements $y_{0}, \ldots, y_{j-1}$. In the limit to the continuous time case this may lead to different answers.

The resemblance goes farther, however. Notice from Appendix $\mathrm{C}$ that

$$
\begin{aligned}
& \bar{k}_{12}(t, s)+\underline{k}_{12}(t, s)=\int_{T}^{0} K_{12}(t, s, \sigma) d \sigma \\
& K_{11}(t, \tau, \sigma)=\left(\int_{\sigma}^{T} K_{12}(t, s, \sigma) d \sigma+\bar{k}_{12}(t, s)\right) C_{e}^{*}(s), \quad \sigma<t \\
& \frac{\partial S_{2}(t, \tau, \sigma)}{\partial \sigma} \equiv 0, \sigma \geqslant t \\
& S_{1}(t, \tau, \sigma)=S_{1}(t, \tau, T), t \leqslant \sigma \leqslant T
\end{aligned}
$$

from which it follows that the function $h(s)$ of equation (4.10) satisfies

$$
\begin{aligned}
h(s)= & \int_{0}^{t}\left\{\bar{k}_{12}(t, s)+\int_{\sigma}^{T} K_{12}(t, s, \rho) d \rho\right\} L(t, \tau, \sigma) d \sigma \\
& +\left\{\bar{k}_{11}(t, s)+\int_{t}^{T} K_{11}(t, s, \sigma) d \sigma\right\} S_{p}(t, \tau) .
\end{aligned}
$$

Defining

$$
\begin{aligned}
\Gamma_{p}(t, \sigma) \triangleq & \bar{k}_{12}(t, T)+\int_{t}^{T} \bar{k}_{12}(t, s) d s \\
& \quad+\int_{\sigma}^{T} K_{12}(t, T, \rho) d \rho+\int_{t}^{T} \int_{\sigma}^{T} K_{12}(t, s, \rho) d \rho d s \\
Y_{p}(t) \triangleq & \bar{k}_{11}(t, T)+\int_{t}^{T} \bar{k}_{11}(t, s) d s \\
& +\int_{t}^{T} K_{11}(t, T, \sigma) d \sigma+\int_{t}^{T} \int_{t}^{T} K_{11}(t, s, \sigma) d \sigma d s
\end{aligned}
$$

then $N_{p}(t, \tau)$ in (4.9) can be written as

$$
N_{p}(t, \tau)=-R_{p}^{-1}(t) B_{p}^{*}(t)\left[Y_{p}(t) S_{p}(t, \tau)+\int_{0}^{t} \Gamma_{p}(t, \sigma) L(t, \tau, \sigma) d \sigma\right]
$$

which equals formula (4.6) of [10]. The equality of $\Gamma_{p}(t, \sigma)$ and $Y_{p}(t)$ with respect to the corresponding functions in [10] has neither been proved nor disproved. 


\subsection{Equal Observations}

A direct approach, as used in previous special cases, appeared not to be the most suitable way to proceed. We have, instead, taken a different route. We consider the special case when $C_{p} \equiv C_{e}$ and $G_{p} \equiv G_{e}$ so that both the players make their decisions based on the same observation data. Thus the state evolves according to

$$
\dot{x}(t ; \omega)=A(t) x(t ; \omega)+B_{p}(t) u_{p}(t ; \omega)+B_{e}(t) u_{e}(t ; \omega)+F(t) n(t ; \omega)
$$

and the observation available to both the players is

$$
y(t ; \omega)=C(t) x(t ; \omega)+G(t) n(t ; \omega) .
$$

The criterion is

$$
\begin{aligned}
J= & \frac{1}{2} E\left\{\left\langle x(T ; \omega), Q_{f} x(T ; \omega)\right\rangle+\int_{0}^{T}(\langle x(t ; \omega), Q(t) x(t ; \omega)\rangle\right. \\
& \left.\left.+\left\langle u_{p}(t ; \omega), R_{p}(t) u_{p}(t ; \omega)\right\rangle-\left\langle u_{e}(t ; \omega), R_{e}(t) u_{e}(t ; \omega)\right\rangle\right) d t\right\} .
\end{aligned}
$$

In the class of controls $u_{p}$ and $u_{e}$ taken as linear functionals of the observation, if we define

$$
\hat{x}(t ; \omega)=E(x(t ; \omega) \mid Y(t ; \omega)),
$$

then $\hat{x}(t ; \omega)$ evolves as

$$
\begin{aligned}
\dot{\hat{x}}(t ; \omega)= & A(t) \hat{x}(t ; \omega)+B_{p}(t) u_{p}(t ; \omega) \\
& +B_{e}(t) u_{e}(t ; \omega)+P(t) C^{*}(t) z(t ; \omega)
\end{aligned}
$$

where

$$
\begin{aligned}
\dot{P}(t)= & A(t) P(t)+P(t) A^{*}(t)+F(t) F^{*}(t) \\
- & \left(P(t) C^{*}(t)+F(t) G^{*}(t)\right)\left(G(t) G^{*}(t)\right)^{-1} \\
& \left(C(t) P(t)+G(t) F^{*}(t)\right) \\
z(t ; \omega)= & y(t ; \omega)-C(t) \hat{x}(t ; \omega)
\end{aligned}
$$

and the cost functional can be reexpressed, excluding some terms independent of the controls, as

$$
\begin{array}{r}
\frac{1}{2} E\left\{\left\langle\hat{x}(T ; \omega), Q_{f} \hat{x}(T ; \omega)\right\rangle+\int_{0}^{T}(\langle\hat{x}(t ; \omega), Q(t) \hat{x}(t ; \omega)\rangle\right. \\
\left.\left.+\left\langle u_{p}(t ; \omega), R_{p}(t) u_{p}(t ; \omega)\right\rangle-\left\langle u_{e}(t ; \omega), R_{e}(t) u_{e}(t ; \omega)\right\rangle\right) d t\right\} .
\end{array}
$$


Assume now that $u_{e}(t ; \omega)=M_{e}(t) \hat{x}(t ; \omega)$. Substituting this in (5.5) and (5.6) and converting our problem to one of optimization in Hilbert space as done in section 3 , the feedback solution is given by

$$
\begin{aligned}
u_{p}^{0}(t)= & -R_{p}^{-1}(t) B_{p}^{*}(t) S_{p}(t) \hat{x}(t ; \omega) \\
\dot{S}_{p}(t)= & \left(-M_{e}^{*} R_{e} M_{e}+Q\right)-\left(A+B_{e} M_{e}\right) S_{p}(t)^{*}-S_{p}(t)\left(A+B_{e} M_{e}\right) \\
& +S_{p}(t) B_{p} R_{p}^{-1} B_{p}^{*} S_{p}(t) ; \quad S_{p}(T)=Q_{f} .
\end{aligned}
$$

We now proceed the other way. Assume that $u_{p}(t ; \omega)=M_{p}(t) \hat{x}(t ; \omega)$. If we substitute this in (5.5) and (5.6), the feedback solution is

$$
\begin{aligned}
u_{e}^{0}(t)= & -R_{e}^{-1}(t) B_{e}^{*}(t) S_{e}(t) \hat{x}(t ; \omega) \\
\dot{S}_{e}(t)= & \left(M_{p}^{*} R_{p} M_{p}+Q\right)-\left(A+B_{p} M_{p}\right)^{*} S_{e}(t)-S_{e}(t)\left(A+B_{p} M_{p}\right) \\
& +S_{e}(t) B_{e} R_{e}^{-1} B_{e}^{*} S_{e}(t) ; \quad S_{e}(T)=-Q_{f}
\end{aligned}
$$

and we obtain

$$
M_{e}=-R_{e}^{-1} B_{e}^{*} S_{e}, \quad M_{p}=-R_{p}^{-1} B_{p}^{*} S_{p}
$$

and therefore,

$$
\begin{aligned}
\dot{S}_{p}= & \left(S_{e} B_{e} R_{e}^{-1} B_{e}^{*} S_{e}-Q\right)-A^{*} S_{p}-S_{p} A+S_{e} B_{e} R_{e}^{-1} B_{e}^{*} S_{p} \\
& +S_{p} B_{e} R_{e}^{-1} B_{e}^{*} S_{e}+S_{p} B_{p} R_{p}^{-1} B_{p}^{*} S_{p} ; \quad S_{p}(T)=Q_{f} \\
\dot{S}_{e}= & \left(S_{p} B_{p} R_{p}^{-1} B_{p}^{*} S_{p}+Q\right)-A^{*} S_{e}-S_{e} A+S_{p} B_{p} R_{p}^{-1} B_{p}^{*} S_{e} \\
& +S_{e} B_{p} R_{p}^{-1} B_{p}^{*} S_{p}+S_{e} B_{e} R_{e}^{-1} B_{e}^{*} S_{e} ; \quad S_{e}(T)=-Q_{f} .
\end{aligned}
$$

It follows from (5.11) and (5.12) that $S_{p} \equiv-S_{e}$, which can be verified by substitution. Writing $S_{p}=-S_{e}=S$, we obtain

$$
\begin{aligned}
& u_{p}^{0}(t)=-R_{p}^{-1}(t) B_{p}^{*}(t) S(t) \hat{x}(t ; \omega) \\
& u_{e}^{0}(t)=R_{e}^{-1}(t) B_{e}^{*}(t) S(t) \hat{x}(t ; \omega)
\end{aligned}
$$

where $S$ satisfies the equation

$$
\dot{S}=-A^{*} S-S A-Q+S\left(B_{p} R_{p}^{-1} B_{p}^{*}-B_{e} R_{e}^{-1} B_{e}^{*}\right) S ; \quad S(T)=Q_{f} .
$$

We are finished if we can show that $u_{p}$ and $u_{e}$ are indeed of the forms $M_{p} \hat{x}$ and $M_{e} \hat{x}$ as assumed. 
For this, we first go over to the extended state $\left[\begin{array}{l}\pi \\ \eta\end{array}\right]$. As was done in section 3 , the problem posed can be converted to

$$
\begin{aligned}
\frac{d}{d t}\left[\begin{array}{l}
\pi \\
\eta
\end{array}\right] & =\left[\begin{array}{cc}
\mathscr{Q}_{0}(t) & 0 \\
0 & 0
\end{array}\right]\left[\begin{array}{l}
\pi \\
\eta
\end{array}\right]+\left[\begin{array}{c}
\mathscr{B}_{p}(t) \\
0
\end{array}\right] u_{p}+\left[\begin{array}{c}
\mathscr{B}_{e}(t) \\
0
\end{array}\right] u_{e}+\left[\begin{array}{c}
\mathscr{F}(t) \\
\mathscr{G}(t)
\end{array}\right] n \\
y & =\left[\begin{array}{ll}
\mathcal{C}(t) & 0
\end{array}\right]\left[\begin{array}{l}
\pi \\
\eta
\end{array}\right]+G(t) n
\end{aligned}
$$

with the operator $\mathfrak{Q}_{0}(t)$ explicitly given in Appendix A.

Equation (4.1) shows that in the case of equal observation,

$$
u_{p}^{0}=\mathscr{N}_{p}\left[\begin{array}{l}
\hat{\pi} \\
\hat{\eta}
\end{array}\right] \text { and } u_{e}^{0}=\mathfrak{K}_{e}\left[\begin{array}{l}
\hat{\pi} \\
\hat{\eta}
\end{array}\right]
$$

for appropriate operators $\mathfrak{K}_{p}$ and $\mathfrak{K}_{e}$. We can paraphrase the argument in the first part of this subsection for $\left[\begin{array}{l}\pi \\ \eta\end{array}\right]$ and we obtain

$$
u_{p}^{0}=-R_{p}^{-1}(t)\left[\mathscr{P}_{p}^{*}(t) \quad 0\right] \mathscr{K}(t)\left[\begin{array}{l}
\hat{\pi}(t ; \omega) \\
\hat{\eta}(t ; \omega)
\end{array}\right]
$$

where

$$
\begin{aligned}
\mathscr{K}(t)= & -\left[\begin{array}{cc}
Q_{0}^{*}(t) & 0 \\
0 & 0
\end{array}\right] \mathscr{K}(t)-\mathscr{K}(t)\left[\begin{array}{cc}
\mathscr{Q}_{0}(t) & 0 \\
0 & 0
\end{array}\right]-\left[\begin{array}{cc}
\chi^{*}(t) \chi(t) & 0 \\
0 & 0
\end{array}\right] \\
& +\mathscr{K}(t)\left(\left[\begin{array}{cc}
\mathscr{B}_{p} R_{p}^{-1} \mathscr{B}_{p}^{*} & 0 \\
0 & 0
\end{array}\right]-\left[\begin{array}{cc}
\mathscr{B}_{e} R_{e}^{-1} \mathscr{B}_{e}^{*} & 0 \\
0 & 0
\end{array}\right]\right) \mathscr{K}(t), \\
\mathscr{K}(T)= & {\left[\begin{array}{cc}
\chi_{f}^{*} \chi_{f} & 0 \\
0 & 0
\end{array}\right] . }
\end{aligned}
$$

The homogeneous equation for $\mathscr{K}_{12}(t)$ implies that $\mathcal{K}_{12} \equiv 0$, and so

$$
u_{p}^{0}(t)=-R_{p}^{-1}(t) \mathscr{G}_{p}^{*}(t) \mathscr{K}_{11}(t) \hat{\pi}(t ; \omega)
$$

Now it follows by definition that $[\hat{\pi}(t ; \omega)](s)=\hat{x}(t ; \omega)$ for $s \geqslant t$ and $\mathscr{K}_{11}(t)$ is an operator acting on a function from $t$ to $T$. Therefore,

$$
u_{p}^{0}(t)=M_{p} \hat{x}
$$

as we assumed in the beginning. This completes our discussion of the equal observation case. 


\section{The Existence of a Solution and the Numerical Procedure}

In this section the existence of $N_{p, e}(t, \tau)$ will be proved, when certain conditions on $\Sigma$ (see (2.8)) are satisfied. A numerical scheme to obtain these functions will be given.

For the sake of simplicity, it is assumed that system noise and observation noises are independent, i.e., $F G_{p}^{*}=0, F G_{e}^{*}=0, G_{e} G_{p}^{*}=0$ and also that $G_{p}, G_{e}, C_{p}$ and $C_{e}$ are time-independent. It will be shown that for $\left\|R_{p}^{-1}\right\|$ and $\left\|R_{e}^{-1}\right\|$ sufficiently small the solution $N_{p, e}$ exists and can be obtained by the repeated application of a contraction operator. From equation (4.7) it follows that

$$
N_{p}(t, \tau)=-R_{p}^{-1}(t)\left[\mathscr{H}_{p}^{*}(t): 0\right] \mathscr{K}_{p}(t) \S_{p}(t, \tau),
$$

where the operators $\mathscr{K}$ and $\mathcal{S}$ have been supplied with the subscript $p$. This subscript has been used because $\mathcal{K}$ and $\delta$ appeared in the derivation of $N_{p}$. Note, however, that this $\mathscr{K}$ and $\mathcal{S}$ do not depend on $N_{p}$; they do depend on $N_{e}$ ! In the same way, an equation for $N_{e}$ exists:

$$
N_{e}(t, \tau)=-R_{e}^{-1}(t)\left[\mathscr{B}_{e}^{*}(t): 0\right] \mathscr{K}_{e}(t) \Im_{e}(t, \tau),
$$

where the meaning of the operators $\mathscr{K}_{e}$ and $\S_{e}$ is clear. Define

$$
\bar{N}_{p, e}(t, \tau)=R_{p, e}(t) N_{p, e}(t, \tau)
$$

and consider the following set of equations:

$$
\begin{aligned}
& \frac{d}{d \tau} \bar{N}_{p}(t, \tau)=-\left[\mathscr{B}_{p}^{*}(t): 0\right] \mathscr{K}_{p}(t) \frac{\partial}{\partial \tau} \delta_{p}(t, \tau) \\
& \frac{d}{d \tau} \bar{N}_{e}(t, \tau)=-\left[\mathscr{R}_{e}^{*}(t), 0\right] \mathscr{K}_{e}(t) \frac{\partial}{\partial \tau} \delta_{e}(t, \tau) \\
& \bar{N}_{p}(t, 0)=-\left[\mathscr{B}_{p}^{*}(t): 0\right] \mathscr{K}_{p}(t){S_{p}}_{p}(t, 0) \\
& \bar{N}_{e}(t, 0)=-\left[\mathscr{B}_{e}^{*}(t), 0\right] \mathscr{K}_{e}(t) \mathcal{S}_{e}(t, 0)
\end{aligned}
$$

The operator $\mathcal{K}_{p}$ is given by means of the differential equation (4.2). The operator $\delta_{p}(t, 0)$ is given by means of equation (4.5). For the operators $\mathcal{K}_{e}$ and $\delta_{e}(t, 0)$ similar results hold, respectively. The differential equation for $\partial /(\partial \tau) \delta_{p, e}(t, \tau)$ is

$$
\frac{d}{d t}\left(\frac{\partial S_{p}(t, \tau)}{\partial \tau}\right)=\Omega(t) \frac{\partial \Im_{p}(t, \tau)}{\partial \tau}
$$

with initial condition

$$
\left.\frac{\partial S_{p}(t, \tau)}{\partial \tau}\right|_{t=\tau}=-\Omega(\tau) \Gamma(\tau)+\frac{d \Gamma(\tau)}{d \tau} .
$$


Similar expressions hold for $(\partial / \partial \tau) \S_{e}(t, \tau)$. The assumptions on $F, G_{p, e}, C_{p, e}$, stated at the beginning of this section have been used to obtain (6.2).

The operator $\mathscr{P}(t)$, defined in equation (4.3), will also be provided with the subscript $p$. Because the operators which appear in the differential equation for $\mathcal{K}(t)$ and $\mathscr{P}(t)$, equations (4.2) and (4.3), are bounded, the arguments of [1] can be used to show that $\mathscr{K}_{p}(t), \mathscr{P}_{p}(t)$, and therefore also $\mathcal{S}_{p}(\tau)$, exist and are bounded for each $N_{e}(t, \tau) \in L_{2}(\mathcal{D})$, where $\mathscr{D}$ is the triangle $0 \leqslant \tau \leqslant t \leqslant T$. Because the differential equations given in Appendix $\mathrm{C}$ are the explicit representations of the operator differential equations for $\mathscr{K}_{p}(t), \mathscr{P}_{p}(t)$, and $\mathcal{S}_{p}(t)$, the solutions of those differential equations are also guaranteed. Analogous remarks hold for $\mathcal{K}_{e}(t)$, $\mathscr{P}_{e}(t)$, and $\delta_{e}(t)$.

In equation (6.1) the dependence on $N_{p, e}$ comes in via the operators $K_{p, e}$, $(\partial / \partial \tau) \delta_{p, e}$ and $\delta_{p, e}(t, 0)$. In the differential equations for these operators we will write $R_{p, e}^{-1} \bar{N}_{p, e}$ instead of $N_{p, e}, \bar{N}_{p, e}^{*} R_{p, e}^{-1} \bar{N}_{p, e}$ instead of $N_{p, e}^{*} R_{p, e} N_{p, e}$ and thus

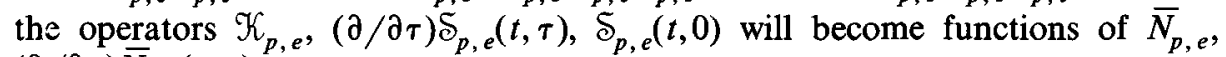
$(\partial / \partial \tau) \bar{N}_{p, e}(t, \tau)$.

Subsequently, terms of the form $\bar{N}_{p, e}(t, \tau)$ in the differential equations for the $\mathcal{F}$ and $\delta$-operators will be replaced by

$$
\bar{N}_{p, e}(t, \tau)=\int_{0}^{\tau} \frac{\partial \bar{N}_{p, e}(t, \sigma)}{\partial \boldsymbol{\sigma}} d \sigma+\bar{N}_{p, e}(t, 0) .
$$

Defining $z \in \mathscr{Z} \triangleq L_{2}[\mathscr{D}] \times L_{2}[\mathscr{D}] \times L_{2}[0, T] \times L_{2}[0, T]$ as

$$
z=\left(z_{1}, z_{2}, z_{3}, z_{4}\right)=\left(\frac{\partial}{\partial \tau} \bar{N}_{p}(t, \tau), \frac{\partial}{\partial \tau} \bar{N}_{e}(t, \tau), \bar{N}_{p}(t, 0), \bar{N}_{e}(t, 0)\right)
$$

we can write equation (6.1) symbolically as

$$
z=\mathscr{K}(z, \mu)
$$

where $\mu=\left(R_{p}^{-1}, R_{e}^{-1}\right)$. The operator $\mathscr{H}$ maps $\mathscr{Z} \times R^{2 n^{2}}$ into $\mathscr{Z}$. For $R_{p}^{-1}=R_{e}^{-1}=0$, i.e., $\mu=0$, the right-hand side of equation (6.3) becomes independent of $z$ because w.r.t. $\bar{N}_{p}, \bar{N}_{e}$ only terms of the form $R_{p, e}^{-1} z_{i}$ and $z_{i}^{*} R_{p, e}^{-1} z_{j}$, to be abbreviated as $a_{p, e i}$ and $b_{p, e i j}$ respectively, appear. Define

$$
z_{0}=\mathcal{K}(., 0) \text {, }
$$

which clearly exists.

Lemma. The operators $\mathcal{K}_{p, e}(t)$ are continuously differentiable w.r.t. $a_{p, e}$ and $b_{p, e}$ $\left[a_{p, e}=\left(a_{p, e 1}, \ldots, a_{p, e 4}\right) ; b_{p, e}\right.$ is similarly defined $]$.

Proof. The right-hand sides of the differential equations for $\mathcal{K}_{p, e}-\mathcal{K}_{p}$ given by equation (4.2)-are continuously differentiable w.r.t. $a_{p . e}$ and $b_{p, e}$. It has already been shown that the operators $\mathscr{K}_{p, e}(t)$ exist on $[0, T]$. The proof of the lemma now directly follows from Theorem 10.7.3 and Remark 10.7.6 in [6]. 
Along the same lines it can be proved that the operators $(\partial / \partial \tau) \S_{p, e}(t, \tau)$ and $\mathfrak{S}_{p, e}(t, 0)$ are continuously differentiable w.r.t. $a_{p, e}$ and $b_{p, e}$. The proof for $(\delta / \partial \tau) \Im_{p, e}(t, \tau)$ is slightly different because, besides the differential equations, the initial conditions also depend on $a_{p, e}, b_{p, e}$. Theorem 10.8.2 of [6] is to be used to take care of this dependence.

Theorem. Let $\mu=\left(R_{p}^{-1}, R_{e}^{-1}\right)$. Then there exists a number $\mu_{0}>0$ and sufficiently small, such that for $\|\mu\| \leqslant \mu_{0}$, the equation

$$
z=\mathscr{H}(z, \mu)
$$

has a unique solution. Furthermore, for $\|\mu\|<\mu_{0}$, the sequence

$$
z_{n+1}=\mathscr{H}\left(z_{n}, \mu\right), \quad n=0,1,2, \ldots
$$

converges to the solution of (6.3), where $z_{0}$ is as defined in (6.4).

Proof. To express the dependence of various operators appearing in $\mathcal{H}$ on $a_{p, e}$, $b_{p, e}$, we explicitly write the various operators as $\mathcal{K}_{p, e}(t, a, b),(\partial / \partial \tau) \mathcal{S}_{p, e}(t, a, b)$ and $\mathcal{S}_{p, e}(t, 0, a, b)$, where $a=\left(a_{p}, a_{e}\right), b=\left(b_{p}, b_{e}\right)$.

Define

$$
v(\mu, z)=\mathscr{K}\left(z+z_{0}, \mu\right)-z_{0} .
$$

Then $v(\mu, 0)=\mathscr{K}\left(z_{0}, \mu\right)-z_{0}$. Now $\mathscr{K}\left(z_{0}, \mu\right)$ is continuous in $\mu$ and the unit ball $\{\mu \mid\|\mu\| \leqslant 1\}$ is compact. It follows that $\left\|\mathscr{K}\left(z_{0}, \mu\right)\right\|$ is bounded for $\mu$ in the unit ball. Therefore, a constant $\beta$ exists such that

$$
\|v(\mu, 0)\| \leqslant \beta / 2 \quad \text { for }\|\mu\| \leqslant 1 .
$$

Let $\mathscr{Z}=\left\{z|| z-z_{0} \|<\beta\right\}$. According to Taylor's formula (Theorem 8.14.3 in [6]), we can write, for any $z$ in $\mathscr{Z}$,

$$
\begin{aligned}
\mathscr{K}_{p, e}(t, a, b)= & \mathscr{K}_{p, e}(t, 0,0)+\frac{\partial \mathscr{K}_{p, e}(t, 0,0)}{\partial a_{p}} a_{p}+\frac{\partial \mathcal{K}_{p, e}(t, 0,0)}{\partial a_{e}} a_{e} \\
& +\frac{\partial \mathcal{K}_{p, e}(t, 0,0)}{\partial b_{p}} b_{p}+\frac{\partial \mathscr{K}_{p, e}(t, 0,0)}{\partial b_{e}} b_{e}+\sigma(\|\mu\|\|z\|) .
\end{aligned}
$$

Analogous expressions hold for $(\partial / \partial \tau) \S_{p, e}(t, \tau, a, b)$, and $\varsigma_{p, e}(t, 0, a, b)$. It follows from (6.8) that a $\mu_{1}>0$ exists such that, for $\|\mu\| \leqslant \mu_{1}$, the operators $\mathscr{K}_{p, e}$, $(\partial / \partial \tau) \delta_{p, e}$, and $\delta_{p, e}$ are bounded; that is, there exists an $M_{1}$ such that $\left\|\mathcal{K}_{p, e}(t, a, b)\right\|,\left\|(\partial / \partial \tau) \delta_{p, e}(t, \tau, a, b)\right\|,\left\|\delta_{p, e}(t, 0, a, b)\right\| \leqslant M_{1}$ for $\|\mu\| \leqslant \mu_{1}$ and any $z$ in $\mathscr{Z}$. Because the operators $\mathscr{B}_{p, e}^{*}$, which also appear in (6.1), are bounded, we can ultimately write

$$
\left\|\mathcal{K}\left(z^{1}, \mu\right)-\mathcal{H}\left(z^{2}, \mu\right)\right\| \leqslant M_{2}\|\mu\|\left\|z^{1}-z^{2}\right\|+\sigma\left(\|\mu\|\left\|z^{1}-z^{2}\right\|\right)
$$


where $M_{2}$ is some finite constant; $z^{1}, z^{2}$ are in $\mathscr{Z}$ and $\|\mu\| \leqslant \mu_{1}$. It follows that we can choose a $\mu_{1}$ such that for $\|\mu\| \leqslant \mu_{1}$,

$$
\left\|\mathscr{H C}\left(z^{1}, \mu\right)-\mathscr{H}\left(z^{2}, \mu\right)\right\| \leqslant \frac{1}{2}\left\|z^{1}-z^{2}\right\|
$$

for $\|\mu\| \leqslant \mu_{1}$ and $z^{1}, z^{2}$ in $\mathcal{Z}$. We translate this inequality to the origin. Let $V$ be the translation of $\mathscr{Z}$ to the origin; that is, $V$ is the open ball defined by $V=\{z \mid\|z\|<\beta\}$. Then, for $z^{1}, z^{2}$ in $V$ and $\|\mu\| \leqslant \mu_{1}$,

$$
\begin{aligned}
\left\|v\left(\mu, z^{1}\right)-v\left(\mu, z^{2}\right)\right\| & =\left\|\mathcal{H}\left(z^{1}+z_{0}, \mu\right)-\mathscr{H}\left(z^{2}+z_{0}, \mu\right)\right\| \\
& =\left\|\mathscr{H}\left(\bar{z}^{1}, \mu\right)-\mathscr{K}\left(\bar{z}^{2}, \mu\right)\right\|, \text { where } \bar{z}^{1}, \bar{z}^{2} \text { are in } \mathscr{Z} \\
& \leqslant \frac{1}{2}\left\|\bar{z}^{1}-\bar{z}^{2}\right\|, \quad \text { by }(6.10) \\
& =\frac{1}{2}\left\|z^{1}-z^{2}\right\| .
\end{aligned}
$$

Let $\mu_{0}>0$ be chosen so that it is less than 1 and $\mu_{1}$. Then (6.11) and (6.7) hold for $\|\mu\|<\mu_{0}$. Let $U=\left\{\mu \mid\|\mu\|<\mu_{0}\right\}$. We can directly apply Theorem 10.1.1 of [6] (identifying $x$ with $\mu, f$ with $z, k=1,2$, and $\alpha$ with $\mu_{0}$ ), which states that there exists a unique mapping $\mu \rightarrow z$ such that

$$
z=v(\mu, z)
$$

for any $\mu$ with $\|\mu\|<\mu_{0}$, and $z(\mu)$ is continuous in $\mu$. Translating back to $z_{0}$, we see that

$$
z+z_{0}=\mathscr{H}\left(z+z_{0}, \mu\right)
$$

has a unique solution, as desired.

The theorem quoted above is constructive and shows directly that the iteration (6.5) converges to the solution of (6.3). This completes our existence result.

Remark. We have proved that, provided certain conditions on $\Sigma$ are satisfied, the optimal $N_{p, e}(t, \tau)$ exist. If the corresponding optimal control functions are denoted by $u_{p}^{*}, u_{e}^{*}$ and the criterion $E\{J\}$ by $V\left(u_{p}, u_{e}\right)$, as was done in section 4 , then

$$
V\left(u_{p}^{*}, u_{e}\right) \leqslant V\left(u_{p}^{*}, u_{e}^{*}\right) \leqslant V\left(u_{p}, u_{e}^{*}\right) .
$$

These inequalities not only hold for linear $u_{e}, u_{p}$, but also for certain nonlinear $u_{p}, u_{e}$, as explained in section 4 . The starting point for the optimal $u_{p}^{*}, u_{e}^{*}$ was the linearity assumption (2.5). Now we are going to relax this assumption and consider the extended class of $u_{p}, u_{e}$ (possibly nonlinear in the observations) such 
that

$$
\begin{aligned}
\dot{x} & =A x+B_{p} u_{p}+B_{e} u_{e}+F n \\
y_{p} & =C_{p} x+G_{p} n \\
y_{e} & =C_{e} x+G_{e} n \\
u_{p} & =\text { nonanticipative functional of } y_{p}(s), \quad 0 \leqslant s<t, \\
u_{e} & =\text { nonanticipative functional of } y_{e}(s), \quad 0 \leqslant s<t,
\end{aligned}
$$

has a unique solution on $[0, T]$. Of course the solution obtained (by means of $N_{p, e}$ ) is also a saddle point in the extended class. Furthermore, it is easily shown that this solution is unique in the extended class. The proof of this statement follows a well-known argument in the theory of zero-sum games [8, p. 13]. Suppose there exists, in this extended class another solution, to be denoted by $\left(\bar{u}_{p}, \bar{u}_{e}\right)$, then also $\left(u_{p}^{*}, \bar{u}_{e}\right)$ and $\left(\bar{u}_{p}, u_{e}^{*}\right)$ are saddle points. However, both $\left(u_{p}^{*}, \bar{u}_{e}\right)$ and $\left(u_{p}^{*}, u_{e}^{*}\right)$ being saddle points yields $\bar{u}_{e}=u_{e}^{*}$.

\section{Conclusion}

We have solved a class of stochastic pursuit-evasion games by converting them to control problems in infinite-dimensional state spaces. A sufficient condition has been given for the existence of saddle point, and an iteration scheme, with the convergence analysis, has been provided for solving the implicit equations that give the kernels for the desired control strategies.

\section{Acknowledgments}

We are indebted to the anonymous referee for his useful comments concerning the initial version of the paper.

\section{Appendix A}

For completeness, we give explicit forms of all operators and their adjoints used in the paper. First we define, for convenience, the operator

$$
I_{s, t}=\begin{array}{lll}
0 & \text { if } \quad s<t \\
I & \text { if } \quad s \geqslant t
\end{array}
$$

where $I$ stands for identity operator on the space of interest. Further, we use the convention

$$
\bar{h}(t)=h(T)+\int_{t}^{T} h(\sigma) d \sigma
$$


whenever $h$ is a function from $[0, T]$ into an appropriate space. Then $\mathbb{Q}(t), \mathscr{P}_{0}: L_{2}^{n}(\mu) \rightarrow L_{2}^{n}(\mu) ; \mathbb{Q}(t) f=g$ or $\mathscr{P}_{0} f=h$ where

$$
\begin{aligned}
& g(s)=I_{s, t}\left[A(t) f(T)+B_{e}(t) \int_{0}^{t} N_{e}(t, \tau) C_{e}(\tau) f(\tau) d \tau\right], \\
& h(s)=P_{0} f(T), \quad 0 \leqslant s \leqslant T .
\end{aligned}
$$

$\mathbb{Q}^{*}(t) h=f$ where

$$
f(s)= \begin{cases}C_{e}^{*}(s) N_{e}^{*}(t, s) B_{e}^{*}(t) \bar{h}(t), & 0<s<t \\ 0, & s=0 \text { or } t \leqslant s<T \\ A^{*}(t) \vec{h}(t), & s=T .\end{cases}
$$

$\mathscr{B}(t): L_{2}^{m_{t}}(\mu) \rightarrow L_{2}^{n}(\mu) ; \Re(t) f=g$ where

$$
g(s)=I_{s, t} B_{e}(t)\left[N_{e}(t, t) f(T)-N_{e}(t, 0) f(0)-\int_{0}^{t}\left(\frac{d}{d \tau} N_{e}(t, \tau)\right) f(\tau) d \tau\right]
$$

$\mathscr{S}^{*}(t) h=f$ where

$$
f(s)= \begin{cases}-N_{e}^{*}(t, 0) B_{e}^{*}(t) \bar{h}(t), & s=0 \\ -\left(\frac{d}{d s} N_{e}^{*}(t, s)\right) B_{e}^{*}(t) \bar{h}(t), & 0<s<t \\ 0, & t \leqslant s<T \\ N_{e}^{*}(t, t) B_{e}^{*}(t) \bar{h}(t), & s=T\end{cases}
$$

$\mathscr{B}_{p}(t): R^{p} \rightarrow L_{2}^{n}(\mu) ; \Re_{p}(t) v=f$ where $f(s)=I_{s, t} B_{p}(t) v$ $\mathscr{S}_{p}^{*}(t) h=x$ where $x=B_{p}^{*}(t) \bar{h}(t)$

$\mathcal{G}_{e}(t): R^{q} \rightarrow L_{2}^{m_{e}}(\mu) ; \mathcal{G}_{e}(t) v=f$ where $f(s)=I_{s, t} G_{e}(t) v$ $\mathcal{G}_{e}^{*}(t) h=x$ where $x=G_{e}^{*}(t) \vec{h}(t)$

$\mathscr{F}(t): R^{q} \rightarrow L_{2}^{n}(\mu) ; \mathscr{F}(t) v=f$ where $f(s)=I_{s, t} F(t) v$ $\mathscr{F}^{*}(t) h=x$ where $x=F^{*}(t) \bar{h}(t)$

$\varrho_{p}(t): L_{2}^{n}(\mu) \rightarrow R^{m_{e}} ; C_{p}(t) f=v$ where $v=C_{p}(t) f(T)$ $\mathcal{C}_{p}^{*}(t) x=h$ where $h(s)=I_{s, T} C_{p}^{*}(t) x$.

$\chi(t), \chi_{f}: L_{2}^{n}(\mu) \rightarrow R^{n} ; \chi(t) f=v$ or $\chi_{f} f=w$ where

$$
v=Q^{\frac{1}{2}}(t) f(T) \quad \text { or } \quad w=Q_{f}^{\frac{1}{2}} f(T) .
$$

$\chi^{*}(t) x=g$ or $\chi_{f}^{*} x=h$ where

$$
g(s)=I_{s, T} Q^{\frac{1}{2}}(t) x \quad \text { or } h(s)=I_{s, T} Q_{f}^{\frac{1}{2}} x
$$


$\mathscr{Q}(t): L_{2}^{n}(\mu) \rightarrow R^{r_{e}} ;$ U $(t) f=v$ where $v=R_{e}^{\frac{1}{2}}(t) \int_{0}^{t} N_{e}(t, \tau) C_{e}(\tau) f(\tau) d \tau$

Q ${ }^{*}(t) x=h$ where

$$
h(s)= \begin{cases}0, s=0 \text { or } & t \leqslant s \leqslant T \\ C_{e}^{*}(s) N_{e}^{*}(t, s) R_{e}^{\frac{r}{2}}(t) x, & 0<s<t\end{cases}
$$

$\checkmark(t): L_{2}^{m_{e}}(\mu) \rightarrow \mathbb{R}^{r_{e}} ; \widetilde{V}(t) f=v$ where

$$
v=R_{e}^{\frac{1}{2}}(t)\left[N_{e}(t, t) f(T)-N_{e}(t, 0) f(0)-\int_{0}^{t}\left(\frac{d}{d \tau} N_{e}(t, \tau)\right) f(\tau) d \tau\right]
$$

$\checkmark *(t) x=h$ where

$$
h(s)= \begin{cases}-N_{e}^{*}(t, 0) R_{e}^{\frac{1}{2}}(t) x, & s=0 \\ -\left(\frac{d}{d s} N_{e}^{*}(t, s)\right) R_{e}^{\frac{1}{2}}(t) x, & 0<s<t \\ 0, & t \leqslant s<T \\ N_{e}^{*}(t, t) R_{e}^{\frac{1}{2}}(t) x, & s=T .\end{cases}
$$

$\mathbb{Q}_{0}(t): L_{2}^{n}(\mu) \rightarrow L_{2}^{n}(\mu) ; \mathbb{Q}_{0}(t) f=g$ where

$$
g(s)=1_{s, t} A(t) f(T) .
$$

\section{Appendix B}

We give here representation of the operators $\mathscr{P}(t)$ and $\mathscr{K}(t)$. We first show that $\Re(t)=E\left[\pi(t ; \omega) \pi^{*}(t ; \omega)\right]$ maps $L_{2}^{n}(\mu)$ into itself and has the structure of an integral operator. From definition, for $f, g$ in $L_{2}^{n}(\mu)$,

$$
E(\langle f, \pi(t ; \omega)\rangle\langle g, \pi(t ; \omega)\rangle)=\langle f, R(t) g\rangle .
$$

Defining $E\left([\pi(t ; \omega)](s)[\pi(t ; \omega)](\tau)^{*}\right)=R(t, s, \tau)$ and using the definition of inner product in $L_{2}^{n}(\mu)$, we have

$$
\begin{aligned}
E(\langle f, \pi(t ; \omega)\rangle\langle g, \pi(t ; \omega)\rangle) \\
=\int_{0}^{T}\left\langle f(s), \int_{0}^{T} R(t, s, \sigma) g(\sigma) d \sigma+R(t, s, T) g(T)+R(t, s, 0) g(0)\right\rangle d s \\
\quad+\left\langle f(T), \int_{0}^{T} R(t, T, \sigma) g(\sigma) d \sigma+R(t, T, T) g(T)+R(t, T, 0) g(0)\right\rangle \\
\quad+\left\langle f(0), \int_{0}^{T} R(t, 0, \sigma) g(\sigma) d \sigma+R(t, 0, T) g(T)+R(t, 0,0) g(0)\right\rangle
\end{aligned}
$$


so that $\mathscr{R}(t) g=h$ where, for $0 \leqslant s \leqslant T$,

$$
h(s)=\int_{0}^{T} R(t, s, \boldsymbol{\sigma}) g(\boldsymbol{\sigma}) d \boldsymbol{\sigma}+R(t, s, T) g(T)+R(t, s, 0) g(0)
$$

Now

$$
\begin{aligned}
\mathscr{P}(t)= & E\left(\left[\begin{array}{c}
\pi(t ; \omega) \\
\eta_{e}(t ; \omega)
\end{array}\right]\left[\pi(t ; \omega) \eta_{e}(t ; \omega)\right]^{*}\right) \\
& -E\left(\left[\begin{array}{c}
\hat{\pi}(t ; \omega) \\
\hat{\eta}_{e}(t ; \omega)
\end{array}\right]\left[\hat{\pi}(t ; \omega) \hat{\eta}_{e}(t ; \omega)\right]^{*}\right) .
\end{aligned}
$$

Extension of the above analysis immediately leads to the fact that $\mathscr{P}(t)$ maps $L_{2}^{n}(\mu) \times L_{2}^{m_{e}}(\mu)$ into itself given by $\mathscr{P}(t) f=g$, where for $0 \leqslant s \leqslant T$,

$$
g(s)=\int_{0}^{T} P(t, s, \sigma) f(\sigma) d \sigma+P(t, s, T) f(T)+P(t, s, 0) f(0)
$$

where the kernel $P$ is a matrix of appropriate order.

To obtain the representation of $\mathscr{K}(t)$, we note that if

$$
\overline{\mathscr{P}}(t)=\mathscr{K}(T-t),
$$

then $\overline{\mathscr{P}}(t)$ is the solution of the same type of Riccati equation as $\mathscr{P}(t) \cdot \overline{\mathscr{P}}(t)$ can, therefore, be interpreted as the covariance operator of filter error of an appropriate dynamical system and has similar integral representation as $\mathscr{P}(t)$, as is explicitly given in equation (4.8).

\section{Appendix C}

Here the equations for the matrices $P, p, \bar{p}, K, \underline{k}, \bar{k}$ and $S$, as introduced in equation (4.8), will be given. These matrices are partitioned as

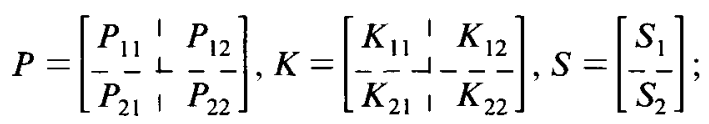

$p, \bar{p}$ are partitioned as $P ; \underline{k}, \bar{k}$ are partitioned as $K$. The sizes of the submatrices are: $P_{11}$ is $n \times n ; P_{12}$ is $n \times m_{e} ; P_{22}$ is $M_{e} \times m_{e} ; P_{21}$ is $m_{e} \times n ; K_{11}$ is $n \times n ; K_{12}$ is $n \times m_{e}$; and so on and $S_{1}$ is $n \times m_{e} ; S_{2}$ is $m_{e} \times m_{e}$. In this appendix the " $t$ " argument for functions of $t$ only will be suppressed. For convenience we will write 
$\tilde{B}_{p, e} \triangleq B_{p, e} R_{p, e}^{-1} B_{p, e}$. The expressions that follow are obtained by straightforward substitution of (4.10) in (4.2), (4.4), and (4.7).

$$
\begin{aligned}
& \frac{\partial S_{1}(t, \tau, s)}{\partial t}=I_{s, t}\left[A S_{1}(t, \tau, T)+B_{e}\left\{\int_{0}^{t} N_{e}(t, \sigma) C_{e}(\sigma) S_{1}(t, \tau, \sigma) d \sigma\right.\right. \\
& +N_{e}(t, t) S_{2}(t, \tau, T)-\int_{0}^{t}\left(\frac{d}{d \sigma} N_{e}(t, \sigma)\right) S_{2}(t, \tau, \sigma) d \sigma \\
& \left.\left.-N_{e}(t, 0) S_{2}(t, \tau, 0)\right\}+B_{p} N_{p}(t, \tau)\right] \\
& -\left[\bar{P}_{11}(t, s) C_{p}^{*}+I_{s, t} F G_{p}^{*}\right]\left(G_{p} G_{p}^{*}\right)^{-1} C_{p} S_{1}(t, \tau, T), \\
& S_{1}(\tau, \tau, s)=\left[\bar{P}_{11}(\tau, s) C_{p}^{*}(\tau)+I_{s, \tau} F(\tau) G_{p}^{*}(\tau)\right]\left(G_{p}(\tau) G_{p}^{*}(\tau)\right)^{-1} ; \\
& \frac{\partial S_{2}(t, \tau, s)}{\partial t}=-\left[\bar{P}_{21}(t, s) C_{p}^{*}+I_{s, t} G_{e} G_{p}^{*}\right]\left(G_{p} G_{p}^{*}\right)^{-1} C_{p} S_{1}(t, \tau, T), \\
& S_{2}(\tau, \tau, s)=\left[\bar{P}_{21}(\tau, s) C_{p}^{*}(\tau)+I_{s, \tau} G_{e}(\tau) G_{p}^{*}(\tau)\right]\left(G_{p}(\tau) G_{p}^{*}(\tau)\right)^{-1} \\
& \frac{\partial P_{11}(t, s, \sigma)}{\partial t}=I_{s, t}\left[A P_{11}(t, T, \sigma)+B_{e}\left\{N_{e}(t, t) P_{21}(t, T, \sigma)-N_{e}(t, 0) P_{21}(t, 0, \sigma)\right.\right. \\
& +\int_{0}^{t} N_{e}(t, \tau) C_{e}(\tau) P_{11}(t, \tau, \sigma) d \tau \\
& \left.\left.-\int_{0}^{t}\left(\frac{d}{d \tau} N_{e}(t, \tau)\right) P_{21}(t, \tau, \sigma) d \tau\right\}\right] \\
& +I_{\sigma, t}\left[\left\{\int_{0}^{t} P_{11}(t, s, \rho) C_{e}^{*}(\rho) N_{e}^{*}(t, \rho) d \rho\right.\right. \\
& +\int_{0}^{t} P_{12}(t, s, \rho) \frac{d}{d \rho} N_{e}(t, \rho) d \rho \\
& \left.\left.+\bar{p}_{12}(t, s) N_{e}^{*}(t, t)+\underline{p}_{12}(t, s) N_{e}^{*}(t, 0)\right\} B_{e}^{*}+\bar{p}_{11}(t, s) A^{*}\right] \\
& +I_{\sigma, t} I_{s, t} F F^{*}-\left(\bar{p}_{11}(t, s) C_{p}^{*}+I_{s, t} F G_{p}^{*}\right)\left(G_{p} G_{p}^{*}\right)^{-1} \\
& \left(C_{p} P_{11}(t, T, \sigma)+I_{\sigma, t} G_{p} F^{*}\right) \text {, }
\end{aligned}
$$

$P_{11}(0, s, \sigma)=P_{0}, \quad 0 \leqslant s, \sigma \leqslant T$ 


$$
\begin{aligned}
& \frac{\partial \bar{p}_{11}(t, s)}{\partial t}=I_{s, t}\left[A \bar{p}_{11}(t, T)+B_{e}\left\{N_{e}(t, t) \bar{p}_{21}(t, T)-N_{e}(t, 0) \bar{p}_{21}(t, 0)\right.\right. \\
& +\int_{0}^{t} N_{e}(t, \rho) C_{e}(\rho) \bar{p}_{11}(t, \rho) d \rho \\
& \left.\left.-\int_{0}^{t}\left(\frac{d}{d \rho} N_{e}(t, \rho)\right) \bar{p}_{21}(t, \rho) d \rho\right\}\right] \\
& +\left\{\int_{0}^{t} P_{11}(t, s, \rho) C_{e}^{*}(\rho) N_{e}^{*}(t, \rho) d \rho\right. \\
& +\int_{0}^{t} P_{12}(t, s, \rho) \frac{d}{d \rho} N_{e}^{*}(t, \rho) d \rho \\
& \left.+\bar{p}_{12}(t, s) N_{e}^{*}(t, t)+\underline{p}_{12}(t, s) N_{e}^{*}(t, 0)\right\} B_{e}^{*} \\
& +\bar{p}_{11}(t, s) A^{*}+I_{s, t} F F^{*}-\left(\bar{p}_{11}(t, s) C_{p}^{*}+I_{s, t} F G_{p}^{*}\right) \\
& \times\left(G_{p} G_{p}^{*}\right)^{-1}\left(C_{p} \bar{p}_{11}(t, T)+G_{p} F^{*}\right), \\
& \frac{\partial \underline{p}_{11}(t, s)}{\partial t}=I_{s, t}\left[\underline{A}_{11}(t, T)\right. \\
& +B_{e}\left\{N_{e}(t, t) \underline{p}_{21}(t, T)+\int_{0}^{t} N_{e}(t, \rho) C_{e}(\rho) \underline{p}_{11}(t, \rho) d \rho\right. \\
& -N_{e}(t, 0) \underline{p}_{21}(t, 0) \\
& \left.\left.-\int_{0}^{t}\left(\frac{d}{d \rho} N_{e}(t, \rho)\right) \underline{p}_{21}(t, \rho) d \rho\right\}\right] \\
& -\left(\bar{p}_{11}(t, s) C_{p}^{*}+I_{s, t} F G_{p}^{*}\right)\left(G_{p} G_{p}^{*}\right)^{-1} C_{p} \underline{p}_{11}(t, T),
\end{aligned}
$$

$\underline{p}_{11}(0, s)=P_{0}, \quad 0 \leqslant s \leqslant T ;$

$$
\begin{array}{rl}
\frac{\partial P_{12}(t, s, \sigma)}{\partial t}=I_{s, t} & A P_{12}(t, T, \sigma)+B_{e}\left\{N_{e}(t, t) P_{22}(t, T, \sigma)\right. \\
& -N_{e}(t, 0) P_{22}(t, 0, \sigma)-\int_{0}^{t}\left(\frac{d}{d \rho} N_{e}(t, \rho)\right) P_{22}(t, \rho, \sigma) d \rho \\
& \left.\left.+\int_{0}^{t} N_{e}(t, \rho) C_{e}(\rho) P_{12}(t, \rho, \sigma) d \rho\right\}\right] \\
& +I_{\sigma, t} I_{s, t} F G_{e}^{*}-\left(\bar{p}_{11}(t, s) C_{p}^{*}+I_{s, t} F G_{p}^{*}\right)\left(G_{p} G_{p}^{*}\right)^{-1} \\
& \times\left(C_{p} P_{12}(t, T, \sigma)+I_{\sigma, t} G_{p} G_{e}^{*}\right),
\end{array}
$$




$$
\begin{aligned}
& P_{12}(0, s, \boldsymbol{\sigma})=0, \quad 0 \leqslant s, \sigma \leqslant T \\
& \frac{\partial \bar{p}_{12}(t, s)}{\partial t}=I_{s, t} {\left[A \bar{p}_{12}(t, T)+B_{e}\left\{N_{e}(t, t) \bar{p}_{22}(t, T)-N_{e}(t, 0) \bar{p}_{22}(t, 0)\right.\right.} \\
&+\int_{0}^{t} N_{e}(t, \rho) C_{e}(\rho) \bar{p}_{12}(t, \rho) d \rho \\
&\left.\left.-\int_{0}^{t}\left(\frac{d}{d \rho} N_{e}(t, \rho)\right) \bar{p}_{22}(t, \rho) d \rho\right\}\right]+I_{s, t} F G_{e}^{*} \\
&-\left(\bar{p}_{21}(t, s) C_{p}^{*}+I_{s, t} F G_{p}^{*}\right)\left(G_{p} G_{p}^{*}\right)^{-1}\left(C_{p} \bar{p}_{12}(t, T)+G_{p} G_{e}^{*}\right),
\end{aligned}
$$

$\bar{p}_{12}(0, s)=0, \quad 0 \leqslant s \leqslant T ;$

$$
\begin{aligned}
\frac{\partial \underline{p}_{12}(t, s)}{\partial t}=I_{s, t}[ & A \underline{p}_{12}(t, T)+B_{e}\left\{N_{e}(t, t) \underline{p}_{22}(t, T)-N_{e}(t, 0) \underline{p}_{22}(t, 0)\right. \\
& \left.\left.+\int_{0}^{t} N_{e}(t, \rho) C_{e}(\rho) \underline{p}_{11}(t, \rho) d \rho-\int_{0}^{t}\left(\frac{d}{d \rho} N_{e}(t, \rho)\right) \underline{p}_{22}(t, \rho) d \rho\right\}\right] \\
& -\left(\bar{p}_{11}(t, s) C_{p}^{*}+I_{s, t} F C_{p}^{*}\right)\left(G_{p} G_{p}^{*}\right)^{-1} C_{p} \underline{p}_{12}(t, T),
\end{aligned}
$$

$\underline{p}_{12}(0, s)=0, \quad 0 \leqslant s \leqslant T ;$

$$
\begin{aligned}
\frac{\partial P_{22}(t, s, \sigma)}{\partial t}= & I_{\sigma, t} I_{s, t} G_{e} G_{e}^{*}-\left(\bar{p}_{21}(t, s) C_{p}^{*}+I_{s, t} G_{e} G_{p}^{*}\right)\left(G_{p} G_{p}^{*}\right)^{-1} \\
& \times\left(C_{p} P_{12}(t, T, \sigma)+I_{\sigma, t} G_{p} G_{e}^{*}\right)
\end{aligned}
$$

$P_{22}(0, s, \sigma)=0, \quad 0 \leqslant s, \sigma \leqslant T$

$$
\begin{aligned}
\frac{\partial \bar{p}_{22}(t, s)}{\partial t}= & I_{s, t} G_{e} G_{e}^{*}-\left(\bar{p}_{21}(t, s) C_{p}^{*}+I_{s, t} G_{e} G_{p}^{*}\right)\left(G_{p} G_{p}^{*}\right)^{-1} \\
& \times\left(C_{p} \bar{p}_{12}(t, T)+I_{\sigma, t} G_{p} G_{e}^{*}\right)
\end{aligned}
$$

$\bar{p}_{22}(0, s)=0, \quad 0 \leqslant s \leqslant T ;$

$$
\frac{\partial \underline{p}_{22}(t, s)}{\partial t}=-\left(\bar{p}_{21}(t, s) C_{p}^{*}+I_{s, t} G_{e} G_{p}^{*}\right)\left(G_{p} G_{p}^{*}\right)^{-1} C_{p} \underline{p}_{12}(t, T)
$$




$$
\begin{aligned}
\underline{p}_{22}(0, s)=0, \quad 0 \leqslant s \leqslant T \\
P_{21}(t, s, \sigma)=P_{12}^{*}(t, s, \sigma) ; \bar{p}_{21}(t, s)=\bar{p}_{12}^{*}(t, s) ; \\
\underline{p}_{21}(t, s)=\underline{p}_{12}^{*}(t, s) ; \\
\frac{\partial K_{11}(t, s, \sigma)}{\partial t}=- \\
\\
\times\left[I_{t, s} C_{e}^{*}(s) N_{e}^{*}(t, s) B_{e}^{*}+I_{s, T} A\right] \\
-I_{t, \sigma}\left[\int_{t}^{T} K_{11}(t, s, \rho) d \rho+\int_{t}^{T} K_{11}(t, \rho, \sigma) d \rho\right] \\
\times B_{e} N_{e}(t, \sigma) C_{e}(\sigma)-I_{t, s} I_{t, \sigma} C_{e}^{*}(s) N_{e}^{*}(t, s) R_{e} N_{e}(t, \sigma) C_{e}(\sigma) \\
+\left[\int_{t}^{T} K_{11}(t, s, \rho) d \rho+\bar{k}_{11}(t, s)\right] \tilde{B}_{p} \\
\times\left[\int_{t}^{T} K_{11}(t, \rho, \sigma) d \rho+K_{11}(t, T, \sigma)\right],
\end{aligned}
$$

$K_{\mathrm{ll}}(T, s, \boldsymbol{\sigma})=0, \quad 0 \leqslant s, \sigma \leqslant T ;$

$$
\begin{aligned}
\frac{\partial \bar{k}_{11}(t, s)}{\partial t}= & -\left[I_{t, s} C_{e}^{*}(s) N_{e}^{*}(t, s) B_{e}^{*}+I_{s, T} A^{*}\right] \\
& \times\left[\bar{k}_{11}(t, T)+\int_{t}^{T} \bar{k}_{11}(t, \rho) d \rho\right] \\
& -\left[\bar{k}_{11}(t, s)+\int_{t}^{T} K_{11}(t, s, \rho) d \rho\right] A \\
& -I_{s, T} Q+\left[\int_{t}^{T} K_{11}(t, s, \rho) d \rho+\bar{k}_{11}(t, s)\right] \\
& \times \tilde{B}_{p}\left[\int_{t}^{T} \bar{k}_{11}(t, \rho) d \rho+\bar{k}_{11}(t, T)\right],
\end{aligned}
$$

$\bar{k}_{11}(T, s)=0, \quad 0 \leqslant s<T ; \quad \bar{k}_{11}(T, T)=Q_{f} ;$

$$
\begin{aligned}
\frac{\partial \underline{k}_{11}(t, s)}{\partial t}= & -\left[I_{t, s} C_{e}^{*}(s) N_{e}^{*}(t, s) B_{e}^{*}+I_{s, T} A^{*}\right]\left[\underline{k}_{11}(t, T)+\int_{t}^{T} \underline{k}_{11}(t, \rho) d \rho\right] \\
& +\left[\int_{t}^{T} K_{11}(t, s, \rho) d \rho+\bar{k}_{11}(t, s)\right] \tilde{B}_{p}\left[\int_{t}^{T} \underline{k}_{11}(t, \rho) d \rho+\underline{k}_{11}(t, T)\right],
\end{aligned}
$$


$\underline{k}_{11}(T, s)=0, \quad 0 \leqslant s \leqslant T$

$$
\begin{aligned}
\frac{\partial K_{12}(t, s, \sigma)}{\partial t}= & -\left[I_{t, s} C_{e}^{*}(s) N_{e}^{*}(t, s) B_{e}^{*}+I_{s, T} A^{*}\right] \\
& \times\left[K_{12}(t, T, \sigma)+\int_{t}^{T} K_{12}(t, \rho, \sigma) d \rho\right] \\
& +I_{t, \sigma}\left[\int_{t}^{T} K_{11}(t, s, \rho) d \rho+\bar{k}_{11}(t, s)+\underline{k}_{11}(t, s)\right] \\
& \times B_{e} \frac{d}{d \sigma} N_{e}(t, \sigma)+I_{t, s} I_{t, \sigma} C_{e}^{*}(s) N_{e}^{*}(t, s) R_{e} \frac{d}{d \sigma} N_{e}(t, \sigma) \\
& +\left[\int_{t}^{T} K_{11}(t, s, \rho) d \rho+\bar{k}_{11}(t, s)\right] \\
& \times \tilde{B}_{p}\left[\int_{t}^{T} K_{12}(t, \rho, \sigma) d \rho+K_{12}(t, T, \sigma)\right],
\end{aligned}
$$

$K_{12}(T, s, \sigma)=0, \quad 0 \leqslant s, \sigma \leqslant T$

$$
\begin{aligned}
\frac{\partial \bar{k}_{12}(t, s)}{\partial t}= & -\left[I_{t, s} C_{e}^{*}(s) N_{e}^{*}(t, s) B_{e}^{*}+I_{s, T} A^{*}\right]\left[\bar{k}_{12}(t, T)+\int_{t}^{T} \bar{k}_{12}(t, \rho) d \rho\right] \\
& -\left[\int_{t}^{T} K_{11}(t, s, \rho) d \rho+\bar{k}_{11}(t, s)+\underline{k}_{11}(t, s)\right] \\
& \times B_{e} N_{e}(t, t)-I_{t, s} C_{e}^{*}(s) N_{e}^{*}(t, s) R_{e} N_{e}(t, t) \\
& +\left[\int_{t}^{T} K_{11}(t, s, \rho) d \rho+\bar{k}_{11}(t, s)\right] \tilde{B_{p}}\left[\int_{t}^{T} \bar{k}_{12}(t, \rho) d \rho+\bar{k}_{12}(t, T)\right]
\end{aligned}
$$

$\bar{k}_{12}(T, s)=0, \quad 0 \leqslant s \leqslant T$

$$
\begin{aligned}
\frac{\partial \underline{k}_{12}(t, s)}{\partial t}= & -\left[I_{t, s} C_{e}^{*}(s) N_{e}^{*}(t, s) B_{e}^{*}+I_{s, T^{A}} A^{*}\right]\left[\underline{k}_{12}(t, T)+\int_{t}^{T} \underline{k}_{12}(t, \rho) d \rho\right] \\
& +\left[\underline{k}_{11}(t, s)+\bar{k}_{11}(t, s)+\int_{t}^{T} K_{11}(t, s, \rho) d \rho\right] B_{e} N_{e}(t, 0) \\
& +I_{t, s} C_{e}^{*}(s) N_{e}^{*}(t, s) R_{e} N_{e}(t, 0) \\
& +\left[\int_{t}^{T} K_{11}(t, s, \rho) d \rho+\bar{k}_{11}(t, s)\right] \tilde{B}_{p}\left[\int_{t}^{T} \underline{k}_{11}(t, \rho) d \rho+\underline{k}_{11}(t, T)\right],
\end{aligned}
$$

$\underline{k}_{12}(T, s)=0, \quad 0 \leqslant s \leqslant T$

$K_{21}(t, s, \sigma) \equiv K_{12}^{*}(t, s, \sigma) ; \bar{k}_{21}(t, s) \equiv \bar{k}_{12}^{*}(t, s) ; \underline{k}_{21}(t, s) \equiv \underline{k}_{12}^{*}(t, s)$. 


\section{References}

1. A. V. Balakrishnan, Stochastic Differential Systems 1, in: Lecture Notes in Economics and Mathematical Systems. No. 84, Springer-Verlag, Berlin, 1973.

2. A. V. Balakrishnan, Applied Functional Analysis. Springer-Verlag, Berlin, 1976.

3. T. Basar, On the uniqueness of the Nash solution in linear-quadratic differential games, International J. Game Theory 5:2/3, 65-90 (1976).

4. R. D. Behn and Y. C. Ho, On a class of linear stochastic differential games, IEEE Transactions on Automatic Control, AC-13, No. 3, 227-239 (June 1968).

5. K. C. Chu and Y. C. Ho, On the generalized linear-quadratic-Gaussian problem, in: H. W. Kuhn, G. P. Szego (eds.), Differential Games and Related Topics, North Holland, Amsterdam, 1971.

6. J. Dieudonné, Foundations of Modern Analysis. Academic Press, New York, 1969.

7. Y. C. Ho and K. C. Chu, Information structure in dynamic multi-person control problems, Automatica 10:4, 341-351 (July 1974).

8. G. Owen, Game Theory, Saunders, Philadelphia, 1968.

9. I. B. Rhodes and D. G. Luenberger, Differential games with imperfect state information, IEEE Transactions on Automatic Control, AC-14, No. 1, 29-38 (February 1969).

10. W. W. Willman, Formal solutions for a class of stochastic-pursuit-evasion games, IEEE Transactions on Automatic Control, AC-14, No. 5, 504-509 (October 1969).

11. N. Ya. Vilenkin et al., Functional Analysis, Wolters-Noordhoff Publishing, Groningen, The Netherlands, 1972.

Received May 20, 1977; accepted for publication January 29, 1979 